\title{
Global Hotspots and Prospects of I-I3I Therapy in Thyroid Carcinoma via Bibliometric Analysis
}

\author{
Shang Lin' \\ Ya-ru Wei ${ }^{2}$ \\ Hong-xiang $\mathrm{Yao}^{3}$ \\ 'Department of Nuclear Medicine, The \\ Second Affiliated Hospital and Yuying \\ Children's Hospital of Wenzhou Medical \\ University, Wenzhou, Zhejiang, 325002, \\ People's Republic of China; ${ }^{2}$ Department \\ of Radiology, The Second Affiliated \\ Hospital and Yuying Children's Hospital \\ of Wenzhou Medical University, \\ Wenzhou, Zhejiang, 325002, People's \\ Republic of China; ${ }^{3}$ Department of \\ Interventional Radiology, The Second \\ Affiliated Hospital and Yuying Children's \\ Hospital of Wenzhou Medical University, \\ Wenzhou, Zhejiang, 325002, People's \\ Republic of China
}

Correspondence: Hong-xiang Yao Department of Interventional Radiology, The Second Affiliated Hospital and Yuying Children's Hospital of Wenzhou Medical University, 109 Xueyuan Western Road, Wenzhou, 325002, Zhejiang, People's Republic of China

Email Isyhx2004@I63.com
Background: Hundreds of studies have reported the application of iodine-131 (I-131) in thyroid carcinoma (THCA) in past years. However, the status of research in the field and other related topics have not been investigated. This study aimed to identify the cooperation of authors, countries, and institutions, as well as explore the hot topics and prospects regarding I-131 therapy in THCA based on previous studies.

Methods: Publications from 2010 to 2020 were retrieved from Web of Science Core Collection according to research strategy. Bibliometric analyses were performed using VOSviewer 1.6.15 and CiteSpace 5.7.3 to evaluate and visualize the cooperation network, hot topic, and research frontier.

Results: The number of publications showed a trend of fluctuation between 2010 and 2020 . We identified 1387 publications related to I-131 therapy in THCA, which were published by 1628 institutions from 82 countries. The largest proportion of publications were emanated from the USA, and the majority of papers were published by Thyroid. Shanghai Jiao Tong University of China contributed the most papers. Although many authors participated in the research of this field, high-yield authors were few. Co-occurrence analysis classified keywords into five clusters, including assessment, efficacy measurement, monitoring, hormone regulation, and guidelines of I-131 therapy. The terms "bone marrow dosimetry and time" were among the latest hotspots. The research frontier topic in I-131 therapy focused on the "P53 and anti-Müllerian hormone".

Conclusion: The attention to I-131 therapy in THCA should be increased considerably. It was necessary to construct active co-operations between authors, countries, and institutions to promote the development of this field. Recent researches referred to the timing and dose assessment of I-131 therapy in THCA. Future studies likely focused on targeted therapy and adverse effects evaluation were worthy of research as well.

Keywords: I-131, thyroid carcinoma, bibliometric analysis, hot spots, research trend

\section{Introduction}

Thyroid carcinoma (THCA) was the most common malignant tumor in endocrine organs, accounting for approximately $1.7 \%$ of total cancer diagnoses. ${ }^{1}$ The treatment choice for THCA included surgery, radiotherapy, and chemotherapy. Thyroidectomy was suitable for the solitary tumors that were freely moveable, but radiotherapy was the treatment of choice for non-resectable canine thyroid carcinoma due to large size or invasion into adjacent structures. ${ }^{2}$ Complete surgical resection was rarely feasible in anaplastic thyroid carcinoma (ATC); therefore, radiation therapy was often used to treat ATC. ${ }^{3}$ Pezzi et al found that radiation therapy dose was associated with improved survival for unresected 
ATC. ${ }^{4}$ Radiotherapy of ATC was often applied in postoperative only, but now it has become a common treatment method in preoperative period. ${ }^{5}$ The ATA recommended that treatment with radioactive iodine can be applied in patients with THCA in T2-T4 or N1 or M1 stage. ${ }^{6}$ Scheffel et al evaluated the impact of the time interval between total thyroidectomy and radioactive iodine administration on disease outcome, and the results found that time interval did not influence outcomes in patients with differentiated thyroid carcinoma. ${ }^{7}$ The previous study has indicated that radiotherapy was beneficial to the survival of patients in particular post-surgical populations. ${ }^{8}$ Currently, hundreds of studies related to I-131 and THCA have been published. However, the status of research in the field and other related topics have not been investigated.

Bibliometric analysis was a statistical method based on the public database to analyze and visualize the research topic and trend. In the present study, we conducted a bibliometric analysis to determine the decade research landscape in terms of the publication year, journals, authors, countries, institutions, keywords, and references, as well as explore the key topics and developments.

\section{Methods}

\section{Data Sources and Search Strategy}

The dataset used in this study was retrieved from the Web of Science Core Collection (WoSCC) on June 01, 2021. The WoSCC is the worldwide largest citation scientific documents database that can help us evaluate the academic importance of papers in a specific field. The search strategy used was as follows: TS=(Thyroid Neoplasms OR Neoplasm, Thyroid OR Thyroid Neoplasm OR Neoplasms, Thyroid OR Thyroid Carcinoma OR Carcinoma, Thyroid OR Carcinomas, Thyroid OR Thyroid Carcinomas OR Cancer of Thyroid OR Thyroid Cancers OR Thyroid Cancer OR Cancer, Thyroid OR Cancers, Thyroid OR Cancer of the Thyroid) AND TS=(Iodine-131 OR I-131 radioisotope OR 131I radioisotope OR $\mathrm{I}-131$ ) $\mathrm{AND} \mathrm{TS}=$ (therapy OR treatment OR disease management) AND publishing year= (2010-2020) AND Language=(English) AND Document types $=$ Article. According to the criteria, 1387 articles written in English between 2010 and 2020 were ultimately included in the final analyses. The full records and references of each publication retrieved in the search, including author names, nationalities, and affiliations, article title, year of publication, name of publishing journal, keywords, and abstract, were downloaded as.txt files from the WoSCC.

\section{Visualization Analysis}

In this study, VOSviewer 1.6.15 was used for constructing and visualizing the bibliometric networks. Collaboration networks were constructed based on co-authorship with a unit of the country, institution, and author, and co-citation analysis associated with the publications. Co-occurrence networks were also constructed to allow visualization of important scientific terms arising from the included publications. In cooperation maps, different nodes represented variant elements. The size of the nodes reflected the number of publications or frequency. The links between nodes represented relationships such as collaboration and co-occurrence. The distance between nodes reflected the association strength. The same color of nodes and lines represented the same clusters.

Besides, the citation burst detection of keywords was performed by Citespace 5.7.3. Citation burst indicated the increased attention to the underlying work over a certain period, which was a key indicator for determining emerging trends. The parameters of CiteSpace were as follows: time slicing (2010-2020), year of the slice, ${ }^{1}$ selection criteria (Top 50), pruning (Pruning sliced networks and Minimum Spanning Tree). In addition, we also used CiteSpace to visualize the research frontier through timezone view.

\section{Results \\ Global Publications}

The number of publications in a specific period reflects the developmental trends of research in a field. We first assessed the publication performance of I-131 therapy in thyroid cancer in 2010-2020 (Figure 1). A total of 1387 articles were published during the past years. These publications have been cited 15,494 times, and each paper was cited 11.17 times on average. The publication output showed an upward trend in 2010-2015. A downward trend was observed in 2015-2019. In 2020, the number of publications increased again. The publications appeared the gyrate up during the past years.

\section{Distribution of Journals}

A total of 1387 articles were published in 394 journals, and Figure 2A presented the collaboration networks between journals. Figure 2B showed the top 10 most popular journals 


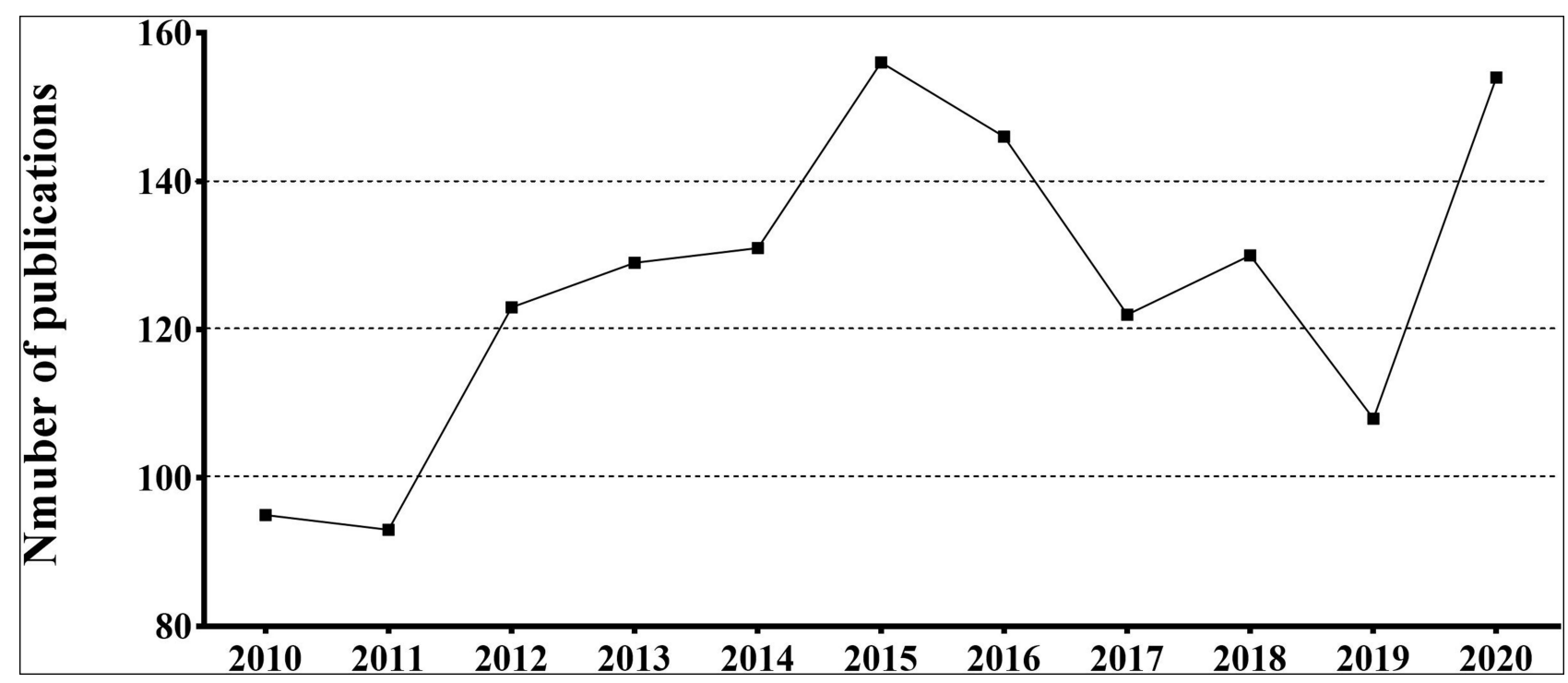

Figure I The annual publication about I-131 therapy in thyroid carcinoma during 2010-2020.

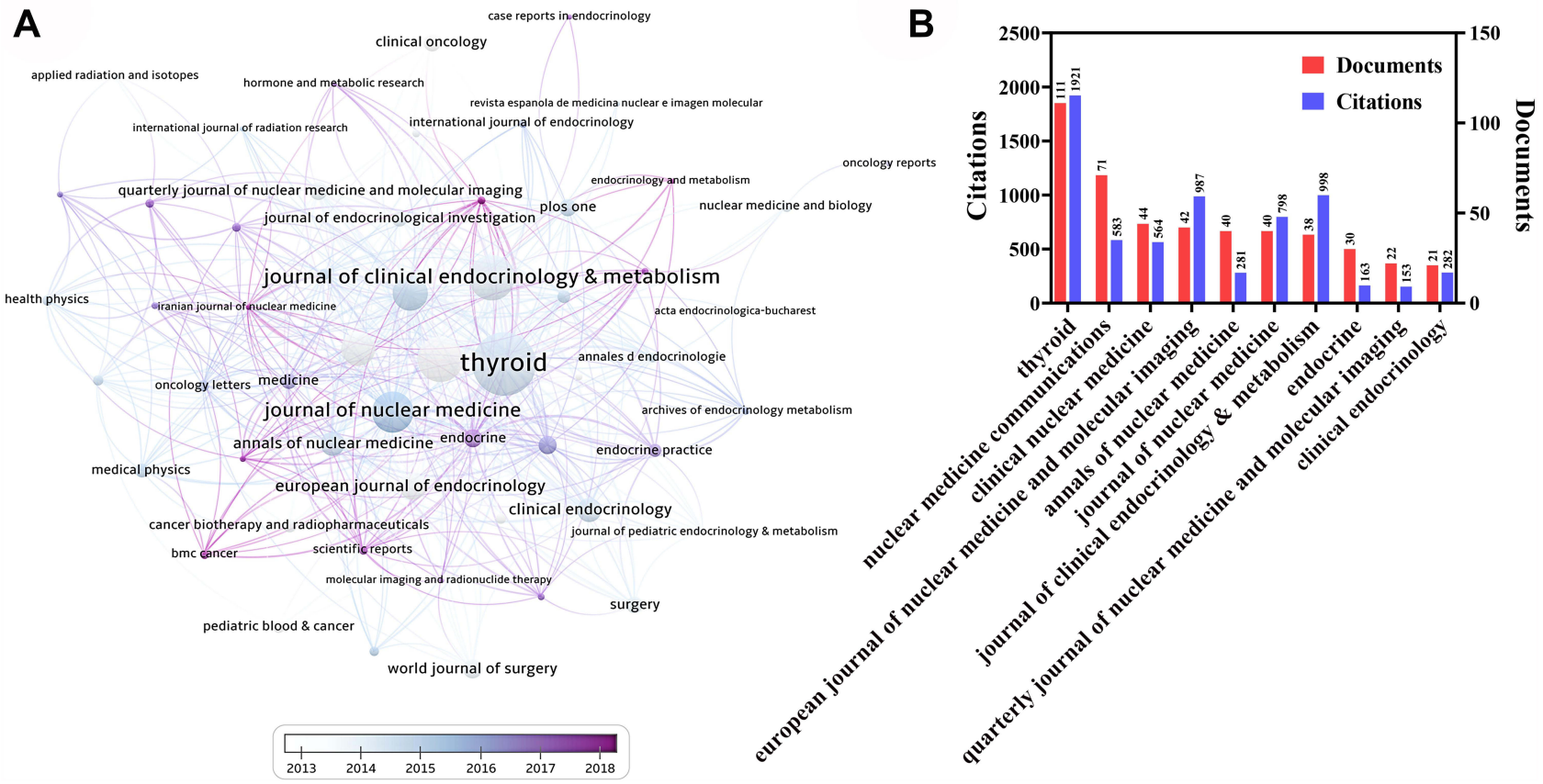

Figure 2 Collaboration network between journals. (A) Productive journals. (B) The top 10 journals.

for publishing articles on I-131 therapy in thyroid cancer. Thyroid published the largest number of articles and showed the largest citations (111 records, 1921 citations, USA), followed by Nuclear Medicine Communications (71, 583, USA), Clinical Nuclear Medicine (44, 564, USA) and European Journal of Nuclear Medicine and Molecular Imaging (42, 987, Germany). Among the top 10 productive journals, 5 journals were from the USA, 2 from the United Kingdom, 1 from Germany, 1 from Japan, and 1 from Italy.

\section{Co-Authorship Analysis in the Unit of Countries, Institutions, and Authors}

Co-authorship maps can help researchers to understand existing partnerships and identify potential partners. We used VOSviewer to analyze the co-authorship between countries, institutions and authors. Figure $3 \mathrm{~A}$ shows the country co-authorship network visualization map. A total of 82 countries participated in the publication of studies on I-131 therapy in thyroid cancer. The top 10 most 
productive countries produced 1112 papers $(80.17 \%)$ of the overall articles. The USA was the foremost productive country with publishing 240 articles, followed by China (217), Italy (117), Germany (115), South Korea (107), Turkey (76), Japan (68), Brazil (65), France (58) and
India. ${ }^{49}$ The publication of the USA was much higher than in other countries. The number of citations was the most commonly utilized tool to determine national efficiency in a specified investigation area. The USA contributed the highest citations (5551), followed by Italy (2880)

\section{A}

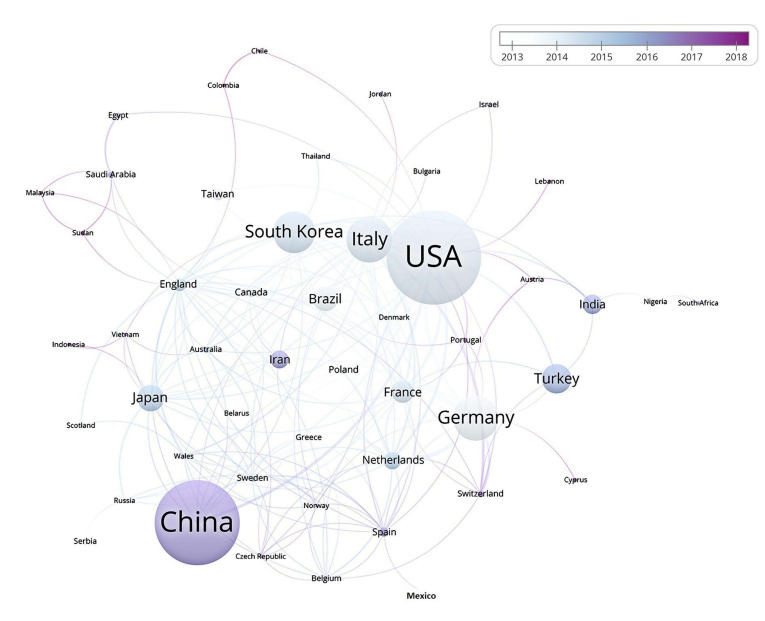

B

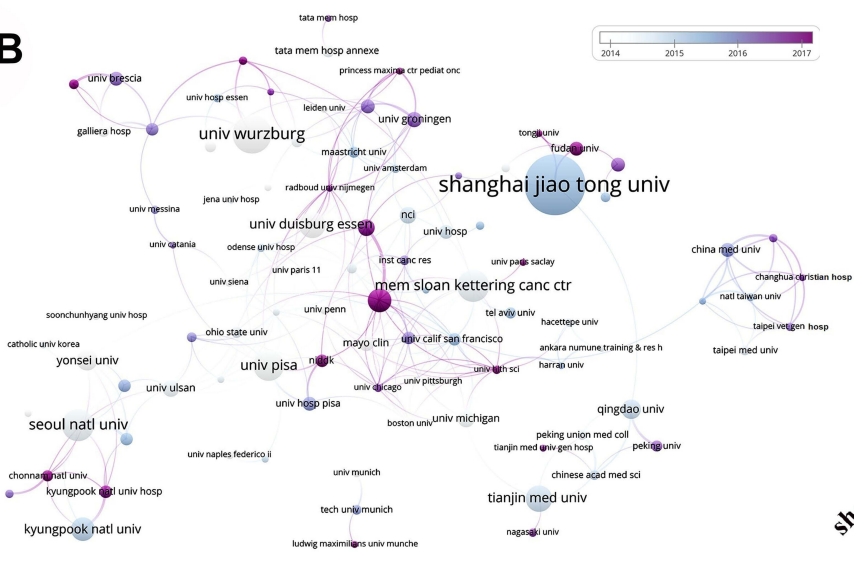

C

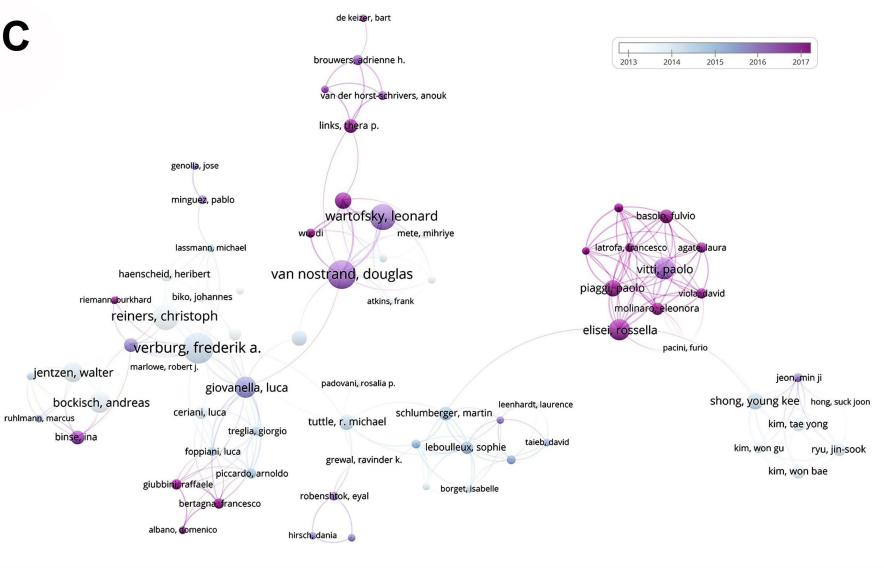

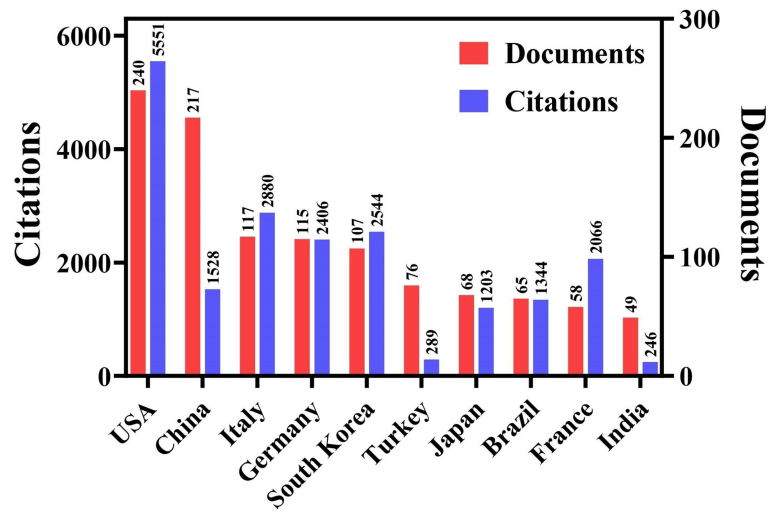
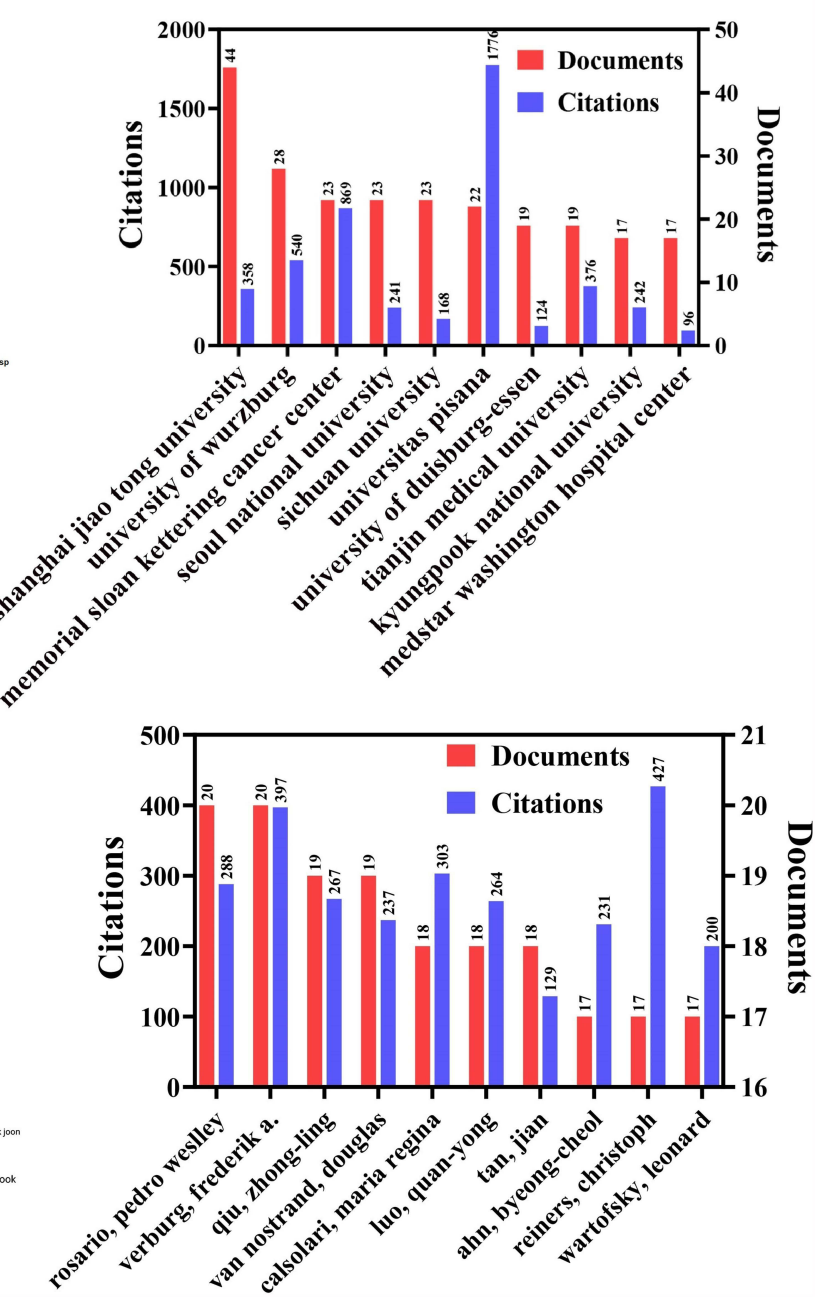

Figure 3 Collaboration network maps. (A) Country. (B) Institution. (C) Author. 
and South Korea (2544). Although the publication number of China ranked second, the citation was far from encouraging.

Figure 3B presents the organization's collaboration network and the top 10 institutions publishing the most articles. A total of 1628 institutions published articles related to I-131 therapy in thyroid cancer. We defined "core institution" as one whose total citation number was higher than 15 , and the total publication number was higher than 5. By this threshold, 121 out of the 1628 organizations were selected. The top three organizations that published higher articles were Shanghai Jiao Tong University (44, China), University of Wurzburg (28, Germany) and Memorial Sloan Kettering Cancer Center (23, USA). University of Texas, MD Anderson Cancer Center ranked the first incited times on average (324.20, USA), followed by University of Pennsylvania (201.43, USA) and Massachusetts General Hospital (179.80, USA).

Figure 3C displays the author's co-authorship network visualization map, and a total of 6580 authors were obtained in the 1387 publications. The top 10 authors contributed $183(13.19 \%)$ papers. The top three authors publishing a higher number of manuscripts were Rosario,
Pedro weslley (20, 1.44\%), Verburg, Frederik a (20, $1.44 \%)$ and Qiu, Zhong-ling (19, 1.37\%).

\section{Co-Cited References Analysis}

For a better understanding of the research contents, we explored the top 10 co-cited references related to I-131 treatment in thyroid cancer research (Table 1). Co-cited references were references that have been co-cited in a set of publications. As observed from Tables 2, 2 articles were co-cited between 200 and 400 times, 5 articles were cocited between 100 and 200 times, and 3 articles were cocited between 90 and 100 times. The latest researches (NO.2) with most co-cited times indicated that recent studies paid more attention to manage patients with radioactive iodine treatment. In addition, assessing the benefits and effectiveness of radioiodine treatment was also the focus of scholars regarding the different doses (NO.6, NO.8). These researches are considered the base of the area due to the high co-citation and great impact.

\section{Co-Occurrence Analysis of Keywords}

Keyword co-occurrence analysis can display the hot spots of research, and keywords with high frequency are often

Table I Top 10 Co-Cited References Related to I-I3I Therapy in Thyroid Cancer

\begin{tabular}{|c|c|c|}
\hline No. & Co-Cited Reference & Co-Citation \\
\hline I & $\begin{array}{l}\text { Revised American Thyroid Association management guidelines for patients with thyroid nodules and differentiated } \\
\text { thyroid cancer. (Cooper DS, Thyroid 2009) }\end{array}$ & 370 \\
\hline 2 & $\begin{array}{l}2015 \text { American Thyroid Association management guidelines for adult patients } \\
\text { with thyroid nodules and differentiated thyroid cancer (Haugen BR, Thyroid 2016) }\end{array}$ & 257 \\
\hline 3 & $\begin{array}{l}\text { European consensus for the management of patients with differentiated thyroid carcinoma of the follicular epithelium. } \\
\text { (Pacini F, Eur J Endocrinol 2006) }\end{array}$ & 151 \\
\hline 4 & $\begin{array}{l}\text { Guidelines for radioiodine therapy of differentiated thyroid cancer. } \\
\text { (Luster M, Eur J Nucl Med Mol Imag 2008)"1 }\end{array}$ & 124 \\
\hline 5 & $\begin{array}{l}\text { Current approaches to primary therapy for papillary and follicular thyroid cancer. (Mazzaferri EL, J Clin Endocr } \\
\text { Metab 200I) }\end{array}$ & 117 \\
\hline 6 & Strategies of radioiodine ablation in patients with low-risk thyroid cancer (Schlumberger $M, N E n g l J M e d 2012)^{13}$ & 110 \\
\hline 7 & $\begin{array}{l}\text { Long-term outcome of } 444 \text { patients with distant metastases from papillary and follicular thyroid carcinoma: benefits and } \\
\text { limits of radioiodine therapy. (Durante C, J Clin Endocrinol Metab 2006) }\end{array}$ & 109 \\
\hline 8 & Ablation with low-dose radioiodine and thyrotropin alfa in thyroid cancer. (Mallick $U, N$ Engl J Med 20I2) ${ }^{15}$ & 99 \\
\hline 9 & Papillary and follicular thyroid carcinoma. (Schlumberger MJ, N Engl J Med 1998) ${ }^{16}$ & 96 \\
\hline 10 & $\begin{array}{l}\text { Long-term impact of initial surgical and medical therapy on papillary and follicular thyroid cancer. (Mazzaferri EL, Am } \\
\text { J Med 1994) }{ }^{17}\end{array}$ & 91 \\
\hline
\end{tabular}


Table 2 The Top 20 Keywords in Terms of Frequency for I- I3I Therapy in Thyroid Cancer Research

\begin{tabular}{|l|l|c|c|l|c|}
\hline Rank & Keywords & Co-Occurrences & Rank & Keywords & Co-Occurrences \\
\hline 1 & Carcinoma & 497 & 11 & I-131 therapy & 119 \\
\hline 2 & Therapy & 309 & 12 & Ablation & 107 \\
\hline 3 & Cancer & 305 & 13 & Serum thyroglobulin & 101 \\
\hline 4 & Management & 241 & 14 & Risk & 99 \\
\hline 5 & Papillary & 217 & 15 & Expression & 90 \\
\hline 6 & I-131 & 203 & 16 & Recurrence & 89 \\
\hline 7 & Radioiodine therapy & 168 & 17 & Radioiodine & 78 \\
\hline 8 & Follow-up & 144 & 18 & Dosimetry & 77 \\
\hline 9 & Radioactive iodine & 126 & 19 & Cancer patients & 70 \\
\hline 10 & Remnant ablation & 124 & 20 & Impact & 69 \\
\hline
\end{tabular}

the main research direction in this field. Co-occurrence analysis helped us to find direction and popular topics in research and has proven to be important in monitoring scientific development. In this study, a total of 2049 keywords appeared, and the top 20 keywords with higher occurrences are presented in Table 2. In addition to main $\mathrm{MeSH}$, the keywords such as management, follow-up, remnant ablation, and serum thyroglobulin showed higher co-occurrence frequency, suggesting that the monitoring and recurrence management of patients draw the attention of scholars.

In co-occurrence analysis of keywords, 50 terms appeared more than or equal to 30 -times and were classified into 5 clusters (Figure 4A). Cluster 1 contained 21 keywords that indicated the I-131 therapy in papillary thyroid carcinoma, frequently mentioned keywords were observed such as ablation, cells, children, disease, dosimetry, expression, and sodium-iodide symporter. Cluster 2 included 12 keywords that referred to the efficacy of I-131 therapy, important keywords such as bone metastases, distant metastases, efficacy, and survival were displayed. Cluster 3 was comprised of eight items that pointed to postoperative monitoring, relevant keywords included follow-up, impact, metastases, positron emission tomography, recurrence, and thyroglobulin. Cluster 4 has seven keywords that correlated with postoperative hormone regulation, and primary keywords were hormone withdrawal, recombinant human thyrotropin and stimulating hormone. Cluster 5 comprised of two keywords that pointed to the guideline of I-131 therapy, the main keywords included association and guidelines.

The overlay visualization map (Figure 4B) scaled by occurrences showed the hotspots in the field of I-131 treatment in thyroid cancer. VOSviewer applied different colors to keywords based on the year that keywords appeared in the literature. Keywords in blue appeared early, followed by red colors, which appeared later. The latest keywords in 2016 included guidelines, recurrence, association, survival, quality of life, and nodules.

CiteSpace was used to detect the burst keywords during the past years, which were identified as indicators of emerging trends. Strong citation bursts can reveal hot words at the forefront of research. The top 24 keywords with the strongest citation bursts during the past decade were shown in Figure 5. Before 2015, researchers focused on the assessment of I-131 therapy, important keywords contained incremental value, consensus, human thyroglobulin, impact, and low-risk patients. During 2015-2020, the researchers paid more attention to the management of patients and relevant keywords included postoperative ablation, bone marrow dosimetry, and time.

Meanwhile, we explored the research trend during the past years (Figure 6). The analysis results of keywords development over time showed that research on I-131 in the thyroid carcinoma in 2020 mainly focused on the following aspects: anti-Müllerian hormone (AMH), P53, accuracy, hyperthyroidism, and hypothyroidism. 


\section{Discussion}

In the current study, we used bibliometric and visualized analyses to explore the research trends and hotspots in the field of I-131 therapy in thyroid carcinoma from 2010 to 2020. It followed that the publications on this topic increased in wave during 2010-2020, and the research topics were relatively diverse. The present study analyzed related articles about this topic from multiple dimensions, showed a systematic view in understanding in this field over the past years and provided guidance for future studies. With the help of our bibliometric analysis, new researchers in this field can easily obtain useful and relevant information.

A total of 1387 articles were published by 1628 institutions from 82 countries. The USA was the leading as it contributed the largest number of papers and citations. Although the publication of China ranked second, China dropped out of the top five when quantifying the significance of citations compared to other countries. In addition, 3 institutions among the top 10 productive institutions were from China, but the citations were also discouraging. This suggested that Chinese researchers are quite interested in this topic; however, quality and influence of their research still needs improvement.

Keyword co-occurrence can accurately reveal the main topic of a field. In our study, 2049 keywords appeared, but only 50 keywords co-occurred greater than 30 times. Cluster analysis classified the 50 terms into 5 clusters. Cluster 1 was mainly related to the I-131 therapy in papillary thyroid carcinoma (PTC). A prospective study by Mourão et al suggested that patients with PTC who have low non-stimulated $\mathrm{Tg}$ and negative neck ultrasonography after thyroidectomy did not require ablation with I-131. ${ }^{18}$ Shang et al found that patients with PTC after total thyroidectomy with low pre-treatment sTg level and few lymph node metastases were more likely to be cured by the first I-treatment. ${ }^{19} \mathrm{Wu}$ et al indicated that patients with PTC with a small size of metastatic lymph node, younger patients, lower thyroglobulin levels, and lymph node metastases detectable on the first post ablative I RxWBS showed a good response to radioiodine ablation therapy. ${ }^{20}$ Cluster 2 mainly focused on the efficacy measurement of I-131 therapy. Rosário indicated that the activity of $1.1 \mathrm{GBq}(30 \mathrm{mCi}) \mathrm{I}-131$ was effective in thyroid ablation in patients with stage T3 and/or N1 PTC and decreased the risk for recurrence. ${ }^{21} \mathrm{Ge}$ et al found that BRAF V600E mutation was related to serum Tg elevation after treatment with I-131 in PTC, indicating that BRAF mutation may be a predictor of the efficacy of I-131 treatment for PTC. ${ }^{22}$ Cluster 3 was related to postoperative monitoring. Lupoli et al concluded that the prediction of disease severity cannot be based exclusively on lesion diameter, a more careful therapeutic approach and followup should be recommended in patients with differentiated thyroid microcarcinoma. ${ }^{23}$ Hung et al suggested that F18fluorodeoxyglucose positron emission tomography can be used to detect metastatic PTC with elevated human serum thyroglobulin levels but negative I-131 whole body scan. ${ }^{24}$
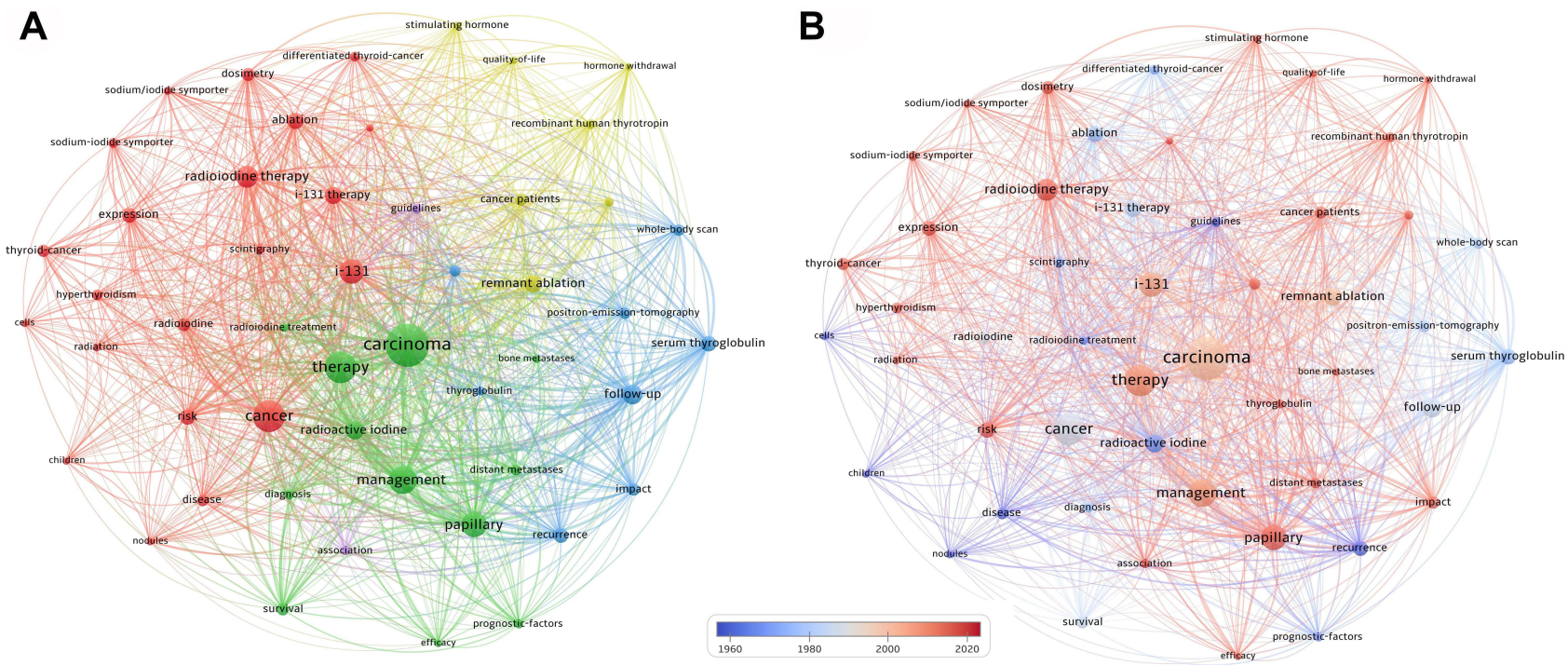

Figure 4 The keywords co-occurrence network. (A) Network visualization. The minimum number of occurrences of a keyword=30. Each color represented a cluster of the keyword. red: Cluster I $(n=21)$, green: Cluster $2(n=12)$, blue: Cluster $3(n=8)$, yellow: Cluster $4(n=7)$, purple: Cluster $5(n=2)$. (B) Overlay visualization. 


\section{Keywords}

incremental value

low risk patient

ct

consensus

human thyrotropin

tumor

radiation

impact

medullary thyroid cancer

i 131 spect/ct

lymph node

ultrasonographic differentiation

thyroid carcinoma

thyrotropin

release

double blind

radioiodineablation

meta-analysis

time

postoperative ablation

iodine radioisotope

association management guideline

bone marrow dosimetry

adult patient
Strength Begin-End

$4.80 \quad 2010-2015$

$4.41 \quad 2010-2015$

$3.67 \quad 2010-2012$

3.65 2010-2015

3.63 2010-2012

$3.41 \quad 2010-2015$

$3.36 \quad 2010-2012$

$3.24 \quad 2010-2012$

2.75 2010-2012

$2.68 \quad 2010-2015$

$6.13 \quad 2013-2018$

$4.12 \quad 2013-2018$

$3.71 \quad 2013-2015$

3.64 2013-2018

$3.30 \quad 2013-2015$

$3.08 \quad 2013-2015$

$2.88 \quad 2013-2015$

$2.86 \quad 2013-2020$

$4.83 \quad 2016-2020$

$3.70 \quad 2016-2018$

$3.50 \quad 2016-2018$

$3.32 \quad 2016-2020$

3.29 2016-2018

$3.17 \quad 2016-2020$
$2010-2020$
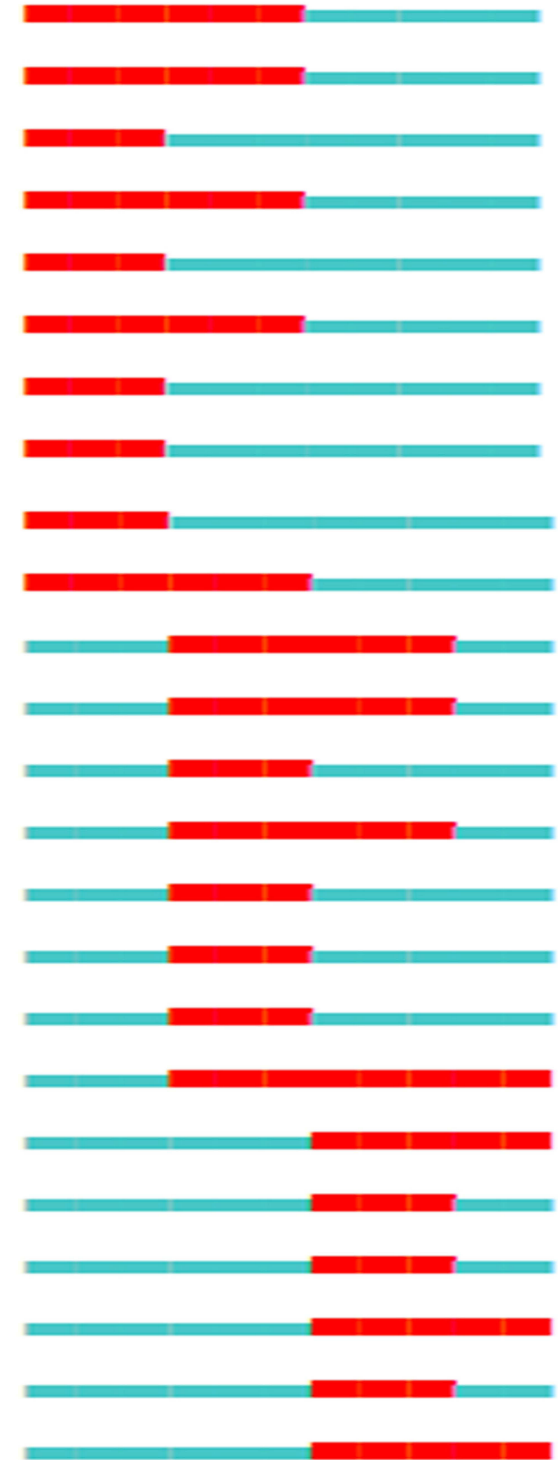

Figure 5 Top 24 keywords with the strongest citation bursts. The red bars meant the keywords occurred frequently. A greater strength indicated a higher frequency of occurrence.

Abbreviations: I-I3I, lodine-I3I; CT, computed tomography.

Locally ablative treatment by radiation therapy was evolving and can be used in selected patients. ${ }^{25}$ In addition, I-131 treatment might cause some potential unfavorable impacts associated with human organs, such as eye, salivary, pulmonary, hematopoietic, and secondary primary malignancies. ${ }^{26}$ Cluster 4 mainly correlated with postoperative hormone regulation. Kalevi et al found that recombinant human TSH stimulation (rhTSH) and thyroid hormone withdrawal (THW) could influence the I-131 biokinetics in patients with differentiated thyroid cancer, and washout of I-131 was faster after rhTSH stimulation than THW. ${ }^{27}$ Kasner et al first found that the combination of rhTSH stimulation and nearly adequate withdrawal may help convert more I-131-negative whole-body scans to positive. ${ }^{28}$ Cluster 5 was mainly concerned about management guidelines. It followed that the clusters of keywords occurrence represented the different aspects of I-131 therapy in thyroid carcinoma.

Keyword co-occurrence network reflected the hot topic in a field, while burst keywords represented the frontier topic. The frontier topic demonstrated the current state of development in a field. As suggested by the present study, 


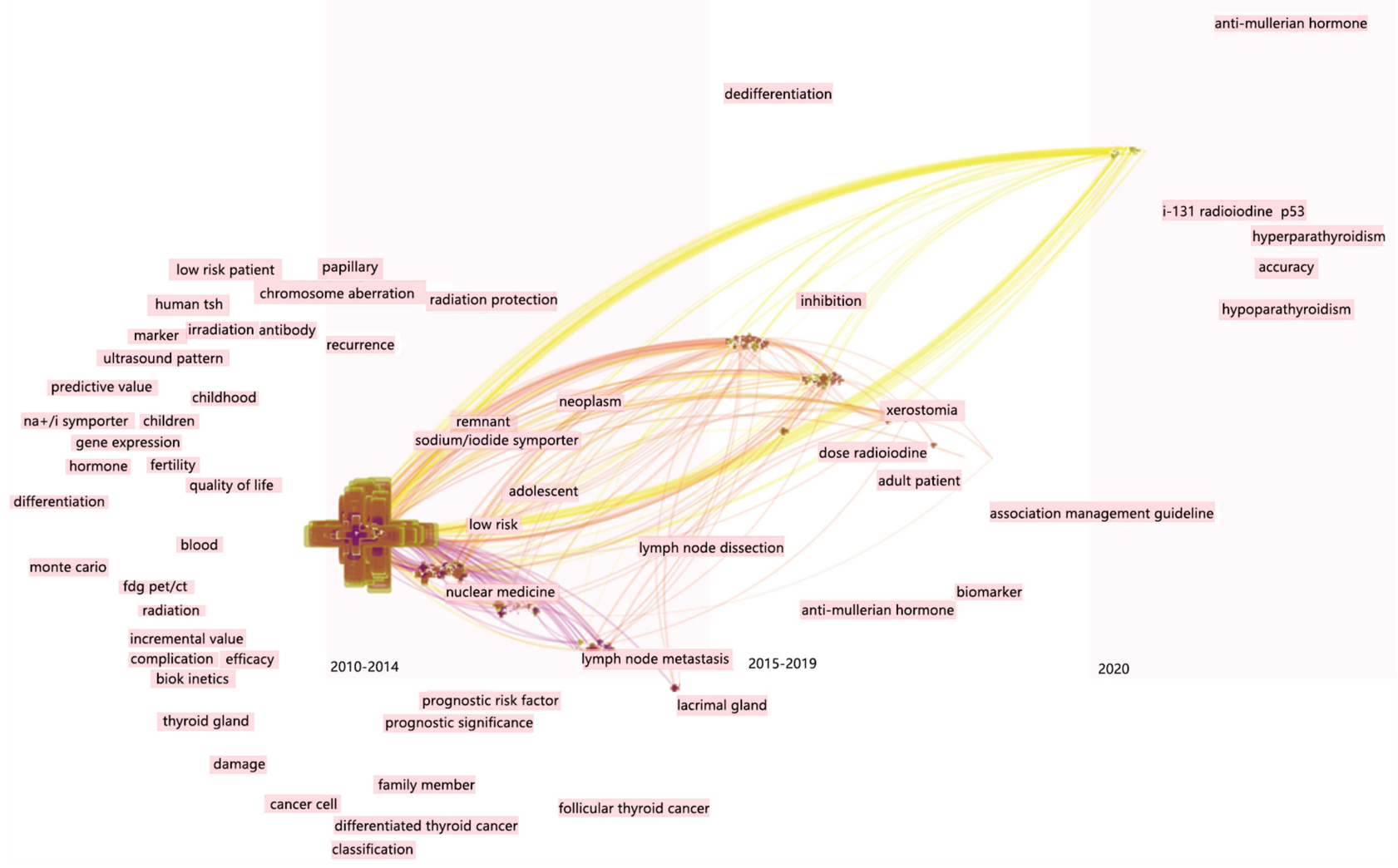

Figure 6 Time-based visualization of keywords indicated the research trend. The "+" represented keywords and larger size indicated a higher occurrence frequency of keywords. The links represented the co-occurrence of keywords.

Abbreviations: I-I3I, lodine-I3I; p53, mutant tumor protein 53; human tsh, human thyroid stimulating hormone; fdg pet, fluorodeoxyglucose positron emission tomography; ct, computed tomography; na+/i symporter, natrium+/iodine symporter.

the main topics focused on the assessment of I-131 therapy before 2015, and relevant keywords included incremental value, impact, and low-risk patients. Zilioli et al demonstrated the incremental diagnostic value of SPECT/CT with I-131 over planar whole bodies scan in patients with differentiated thyroid carcinoma, and SPECT/CT with I-131 can affect therapy decision-making and patient management. ${ }^{29}$ Raruenrom et al found that microscopic positive margin was significantly associated with the incomplete response after I-131 therapy in patients with differentiated thyroid carcinoma after adjusting for tumor size, cervical lymph node metastasis, and pre-ablative Tg. ${ }^{30}$ It followed that the application of I-131 for the treatment of thyroid carcinoma was recognized during this period. After 2015, researches paid more attention to the monitoring and recurrence management associated with I-131. The key issue in I-131 treatment for differentiated thyroid carcinoma was the timing of I-131 therapy. ${ }^{31}$ The dose of I-131 therapy also should not be ignored. Zupunski et al found a significant linear-quadratic doseeffect association between thyroid cancer and iodine thyroid dose in the range up to 5 grays. ${ }^{32}$ Pre-treatment dosimetry before I-131 treatment for patients with advanced differentiated thyroid cancer was conducive to provide critical information for optimizing dosing regimen for effectiveness and safety. ${ }^{33}$ For thyroid remnant ablation after thyroid hormone withdrawal, the efficacy of 2220 MBq I-131 was just as effective as $3700 \mathrm{MBq}$ and exerted fewer long-term side effects. ${ }^{34}$

As indicated from the time-zone view, the latest keywords in 2020 focused on anti-Müllerian hormone (AMH), P53, accuracy, hyperthyroidism, and hypothyroidism. The AMH is an important indicator associated with females and strongly correlated with antral follicle count, which may be a tool to measure fertility status ${ }^{35}$ and good marker for ovarian reserve. Differentiated thyroid cancer occurs more frequent in females than it does in males, and a large proportion of these female patients is in their reproductive years. ${ }^{36}$ Although I-131 treatment resulted in excellent disease-specific survival in young females, ${ }^{37}$ the examination of possible long-term effects on their reproductive function was extremely necessary. In recent years, several 
studies have been conducted to evaluate the effects of RAI therapy on fertility risks in female patients. ${ }^{38-40}$ But the firm conclusion was not obtained, which may be affected by various elements such as research design, sample size, time of follow-up, thyroidectomy method (total/subtotal), and I-131 therapy method (single or multiple). Therefore, it was great importance to conduct more investigations in the future to improve the woman's health.

The TP53 gene (p53) is a tumor suppressor gene and one of the most frequently mutated genes in human cancer. The p53 mutations occurred in $\sim 50 \%$ of tumor cells. ${ }^{41}$ In terms of thyroid carcinoma, mutations in the p53 gene were reported in up to $40 \%$ of PTCs and $22 \%$ of FTCs, and were associated with a worse prognosis. ${ }^{42,43}$ The treatment of thyroid carcinoma targeted p53 might be a valuable method to improve the patient's clinical outcome. In addition, p53 also exerted immune regulatory functions ${ }^{44}$ by suppressing inflammation and autoimmunity. The immunotherapy has become a powerful strategy for patients' treatment aiming to generate potent antitumor responses, but immunotherapies can generate autoimmune responses. A recent study indicated that $\mathrm{p} 53$ reactivation exerted beneficial effects on tumor progression by restoring antitumoral immune responses whilst avoiding the development of autoimmune responses in thyroid carcinoma. ${ }^{45}$ It followed that the targeted treatment associated with p53 in thyroid carcinoma were needed more investigations to improve the patient's health.

Additionally, RAI has been used extensively to treat hyperthyroidism and considered as second-line treatment when unsuccessful therapy with an antithyroid drug (ATD), disease relapse, or adverse reactions to medical therapy occurred. ${ }^{46}$ The RAI treatment is to achieve permanent hypothyroidism, but this is not consistently achieved as many factors were associated with the success of RAI. Kwak et al showed that methimazole use $>3$ months was associated with I-131 treatment failure, ${ }^{47}$ suggesting that the association between long-term first-line ATD use and RAI treatment failure was needed further investigations. Namwongprom et al showed that I-131 dose of $250-400 \mu \mathrm{Ci} / \mathrm{g}$ was suitable in patients with hyperthyroidism with medical failure from ATDs. ${ }^{48}$ And testing at 2-3 months after RAI was most helpful to confirm response to RAI. ${ }^{49}$ These findings reminded us that the effectiveness of I-131 therapy was closely related to the ATD use, and the time of ATD use, dose of I-131 and testing time after I-131 treatment should be noticed in clinical practice.
The present study used bibliometric analysis to explore the I-131 therapy in thyroid cancer. We performed frequency analysis, cluster analysis, and hotspot analysis to clearly show the current status and future trends. However, we only searched the database of Web of Science and included research articles cannot be enough to represent all related literature. In addition, we just introduced the English publications into our analysis, which may cause language bias. It was acknowledged that the present study existed several limitations, it still revealed the future research trends and hotspots in this research field to some extent.

\section{Conclusions}

Taken together, the current study summarized the global research trends concerning I-131 therapy in thyroid carcinoma. Thyroid was the top 1 productive journal. The USA led in this research field with the highest number of publications and citations. Shanghai Jiao Tong University of China did a great deal of research in this field and contributed the largest publications. Active collaborations were observed among the main authors. The main research topic focused on the management of patients such as dose and timing determination of I-131 therapy. Research frontier referred to the targeted therapy associated with p53 and adverse effects assessment of I-131 therapy. These results provided a new perspective for the study of I-131 therapy in thyroid carcinoma, which might have a beneficial effect on the further etiological study, diagnosis, and treatment of thyroid carcinoma.

\section{Data Sharing Statement}

The datasets used and/or analyzed during the current study are available from the corresponding authors upon reasonable request.

\section{Acknowledgement}

Ya-ru Wei is currently affiliated with the School of Laboratory Medicine and Life Sciences, WenZhou Medical University, Wenzhou, Zhejiang, 325002, People's Republic of China.

\section{Funding}

This work was supported by the Clinical Research Special Fund of $\mathrm{Wu}$ Jieping Medical Foundation (No. 320.6750.2020-10-36), and the Medical and Health Science and Technology Project of Zhejiang Province (2019PY048). 


\section{Disclosure}

The authors report no conflicts of interest for this work.

\section{References}

1. He W, Qi B, Zhou Q, et al. Key genes and pathways in thyroid cancer based on gene set enrichment analysis. Oncol Rep. 2013;30 (3):1391-1397. doi:10.3892/or.2013.2557

2. Tsimbas K, Turek M, Christensen N, Vail DM, Forrest L. Short survival time following palliative-intent hypofractionated radiotherapy for non-resectable canine thyroid carcinoma: a retrospective analysis of 20 dogs. Vet Radiol Ultrasound. 2019;60(1):93-99.

3. Nagaiah G, Hossain A, Mooney CJ, Parmentier J, Remick SC. Anaplastic thyroid cancer: a review of epidemiology, pathogenesis, and treatment. J Oncol. 2011;2011:542358.

4. Pezzi TA, Mohamed ASR, Sheu T, et al. Radiation therapy dose is associated with improved survival for unresected anaplastic thyroid carcinoma: outcomes from the National Cancer Data Base. Cancer. 2017;123(9):1653-1661.

5. Are C, Shaha AR. Anaplastic thyroid carcinoma: biology, pathogenesis, prognostic factors, and treatment approaches. Ann Surg Oncol. 2006;13 (4):453-464.

6. American Thyroid Association Guidelines Taskforce on Thyroid N, Differentiated Thyroid C; Cooper DS, Doherty GM, Haugen BR, et al. Revised American Thyroid Association management guidelines for patients with thyroid nodules and differentiated thyroid cancer. Thyroid. 2009;19(11):1167-1214.

7. Scheffel RS, Zanella AB, Dora JM, Maia AL. Timing of radioactive iodine administration does not influence outcomes in patients with differentiated thyroid carcinoma. Thyroid. 2016;26(11):1623-1629.

8. Brierley J, Tsang R, Panzarella T, Bana N. Prognostic factors and the effect of treatment with radioactive iodine and external beam radiation on patients with differentiated thyroid cancer seen at a single institution over 40 years. Clin Endocrinol (Oxf). 2005;63 (4):418-427.

9. Haugen BR, Alexander EK, Bible KC, et al. 2015 American Thyroid Association management guidelines for adult patients with thyroid nodules and differentiated thyroid cancer: the American Thyroid Association guidelines task force on thyroid nodules and differentiated thyroid cancer. Thyroid. 2016;26(1):1-133.

10. Pacini F, Schlumberger M, Dralle H, et al. European consensus for the management of patients with differentiated thyroid carcinoma of the follicular epithelium. Eur J Endocrinol. 2006;154(6):787-803. doi:10.1530/eje.1.02158

11. Luster M, Clarke SE, Dietlein M, et al. Guidelines for radioiodine therapy of differentiated thyroid cancer. Eur J Nucl Med Mol Imaging. 2008;35(10):1941-1959.

12. Mazzaferri EL, Kloos RT. Clinical review 128: current approaches to primary therapy for papillary and follicular thyroid cancer. $J$ Clin Endocrinol Metab. 2001;86(4):1447-1463.

13. Schlumberger M, Catargi B, Borget I, et al. Strategies of radioiodine ablation in patients with low-risk thyroid cancer. $N$ Engl $J$ Med 2012;366(18):1663-1673.

14. Durante C, Haddy N, Baudin E, et al. Long-term outcome of 444 patients with distant metastases from papillary and follicular thyroid carcinoma: benefits and limits of radioiodine therapy. $J$ Clin Endocrinol Metab. 2006;91(8):2892-2899.

15. Mallick U, Harmer C, Yap B, et al. Ablation with low-dose radioiodine and thyrotropin alfa in thyroid cancer. $N$ Engl J Med. 2012;366 (18):1674-1685.

16. Schlumberger MJ. Papillary and follicular thyroid carcinoma. $N$ Engl J Med. 1998;338(5):297-306.

17. Mazzaferri EL, Jhiang SM. Long-term impact of initial surgical and medical therapy on papillary and follicular thyroid cancer. Am J Med. 1994;97(5):418-428.
18. Mourao GF, Rosario PW, Calsolari MR. Low postoperative nonstimulated thyroglobulin as a criterion to spare radioiodine ablation. Endocr Relat Cancer. 2016;23(1):47-52.

19. Shang L, Fang S, Zhang P, et al. Impact factors for the outcome of the first (131)I radiotherapy in patients with papillary thyroid carcinoma after total thyroidectomy. Ann Nucl Med. 2019;33(3):177-183.

20. Wu X, Gu H, Gao Y, Li B, Fan R. Clinical outcomes and prognostic factors of radioiodine ablation therapy for lymph node metastases from papillary thyroid carcinoma. Nucl Med Commun. 2018;39(1):22-27.

21. Rosario PW, Calsolari MR. Thyroid ablation with $1.1 \mathrm{GBq}(30 \mathrm{mCi})$ iodine-131 in patients with papillary thyroid carcinoma at intermediate risk for recurrence. Thyroid. 2014;24(5):826-831.

22. Ge J, Wang J, Wang $\mathrm{H}$, et al. The BRAF V600E mutation is a predictor of the effect of radioiodine therapy in papillary thyroid cancer. J Cancer. 2020;11(4):932-939.

23. Lupoli R, Cacciapuoti M, Tortora A, et al. Clinical outcome in differentiated thyroid carcinoma and microcarcinoma. Int J Surg. 2014;12(Suppl 1):S148-51.

24. Hung $\mathrm{MC}$, Wu HS, Kao $\mathrm{CH}$, Chen WK, Changlai SP. F18fluorodeoxyglucose positron emission tomography in detecting metastatic papillary thyroid carcinoma with elevated human serum thyroglobulin levels but negative I-131 whole body scan. Endocr Res. 2003;29 (2):169-175.

25. Giovanella L, Scappaticcio L. Radioiodine therapy of advanced differentiated thyroid cancer: clinical considerations and multidisciplinary approach. Q J Nucl Med Mol Imaging. 2019;63:229-234.

26. Van Nostrand D. The benefits and risks of I-131 therapy in patients with well-differentiated thyroid cancer. Thyroid. 2009;19(12):1381-1391.

27. Kairemo K, Kangasmaki A, Bom HS. Comparison of I-131 biokinetics after recombinant human TSH stimulation and thyroid hormone withdrawal measured by external detector in patients with differentiated thyroid cancer. Chonnam Med J. 2019;55(1):20-24.

28. Kasner DL, Spieth ME, Starkman ME, Zdor-North D. Thyroid carcinoma: iodine-131-negative whole-body scan reverses to positive after a combination of thyrogen stimulation and withdrawal. Clin Nucl Med. 2002;27(11):772-780.

29. Zilioli V, Peli A, Panarotto MB, et al. Differentiated thyroid carcinoma: incremental diagnostic value of (131)I SPECT/CT over planar whole body scan after radioiodine therapy. Endocrine. 2017;56(3):551-559.

30. Raruenrom Y, Sawangsri K, Somboonporn C, Theerakulpisut D, Wongsurawat N, Ratanaanekchai T. An impact of microscopic positive margin on incomplete response after I-131 treatment in differentiated thyroid cancer. Ann Nucl Med. 2020;34(7):453-459.

31. Smallridge RC, Ain KB, Asa SL, et al. American Thyroid Association guidelines for management of patients with anaplastic thyroid cancer. Thyroid. 2012;22(11):1104-1139.

32. Zupunski L, Ostroumova E, Drozdovitch V, et al. Thyroid cancer after exposure to radioiodine in childhood and adolescence: (131) I-related risk and the role of selected host and environmental factors. Cancers (Basel). 2019;11:10.

33. Chen MK, Cheng DW. What is the role of dosimetry in patients with advanced thyroid cancer? Curr Opin Oncol. 2015;27(1):33-37.

34. Fish SA, Basu S, Alavi A, Mandel SJ. Comparison of efficacy of $2220 \mathrm{MBq}$ versus $3700 \mathrm{MBq} \mathrm{I}-131$ for ablation of thyroid remnant in patients with differentiated thyroid cancer. Quart J Nucl Med Mol Imaging. 2010;54(5):560-563.

35. Broer SL, Broekmans FJM, Laven JSE, et al. Anti-Müllerian hormone: ovarian reserve testing and its potential clinical implications. Hum Reprod Update. 2014;20:688-701.

36. Qian ZJ, Jin MC, Meister KD, Megwalu UC. Pediatric thyroid cancer incidence and mortality trends in the United States, 1973-2013. JAMA Otolaryngol Head Neck Surg. 2019;145(7):617-623.

37. van Velsen EFS, Stegenga MT, van Kemenade FJ, et al. Comparing the prognostic value of the Eighth Edition of the American Joint Committee on Cancer/Tumor Node Metastasis Staging System Between Papillary and Follicular Thyroid Cancer. Thyroid. 2018;28(8):976-981. 
38. Anagnostis P, Florou P, Bosdou JK, et al. Decline in anti-Müllerian hormone concentrations following radioactive iodine treatment in women with differentiated thyroid cancer: a systematic review and meta-analysis. Maturitas. 2021;148:40-45.

39. Piek MW, Postma EL, van Leeuwaarde R, et al. The effect of radioactive iodine therapy on ovarian function and fertility in female thyroid cancer patients: a systematic review and meta-analysis. Thyroid. 2021;31(4):658-668.

40. Nies M, Cantineau AEP, Arts EGJM, et al. Long-term effects of radioiodine treatment on female fertility in survivors of childhood differentiated thyroid carcinoma. Thyroid. 2020;30(8):1169-1176.

41. Bykov Vladimir JN, Eriksson Sofi E, Bianchi J, et al. Targeting mutant p53 for efficient cancer therapy. Nat Rev Cancer. 2018;18:89-102.

42. Bykov VJN, Eriksson SE, Bianchi J, Wiman KG. Targeting mutant p53 for efficient cancer therapy. Nat Rev Cancer. 2018;18(2):89-102.

43. Cancer Genome Atlas Research Network. Integrated genomic characterization of papillary thyroid carcinoma. Cell. 2014;159 (3):676-690.
44. Muñoz-Fontela C, Mandinova A, Aaronson SA, Lee SW. Emerging roles of p53 and other tumour-suppressor genes in immune regulation. Nat Rev Immunol. 2016;16(12):741-750.

45. Arena A, Stigliano A, Belcastro E, et al. p53 activation effect in the balance of $\mathrm{T}$ regulatory and effector cell subsets in patients with thyroid cancer and autoimmunity. Front Immunol. 2021;12:728381.

46. Wartofsky L, Glinoer D, Solomon B, et al. Differences and similarities in the diagnosis and treatment of Graves' disease in Europe, Japan, and the United States. Thyroid. 1991;1(2):129-135.

47. Kwak JJ, Altoos R, Jensen A, Altoos B, McDermott MT. Increased risk of radioiodine treatment failure associated with graves disease refractory to methimazole. Endocr Pract. 2020;26(11):1312-1319.

48. Namwongprom S, Dejkhamron P, Unachak K. Success rate of radioactive iodine treatment for children and adolescent with hyperthyroidism. J Endocrinol Invest. 2021;44(3):541-545.

49. Kaplowitz PB, Jiang J, Vaidyanathan P. Radioactive iodine therapy for pediatric Graves' disease: a single-center experience over a 10year period. J Pediatr Endocrinol Metab. 2020;33(3):383-389.

\section{Publish your work in this journal}

The International Journal of General Medicine is an international, peer-reviewed open-access journal that focuses on general and internal medicine, pathogenesis, epidemiology, diagnosis, monitoring and treatment protocols. The journal is characterized by the rapid reporting of reviews, original research and clinical studies across all disease areas. The manuscript management system is completely online and includes a very quick and fair peer-review system, which is all easy to use. Visit http://www.dovepress.com/ testimonials.php to read real quotes from published authors. 